\title{
Schockraummanagement - von der Alarmierung bis zum interdisziplinären Weiterbehandlungskonzept
}

Chistopher Spering, Stephan Sehmisch, Wolfgang Lehmann

\section{Einleitung}

Der Schockraum stellt die Schnittstelle zwischen präklinischer Initialversorgung und interdisziplinärer innerklinischer Definitivversorgung Schwerstverletzter dar. Insbesondere in einem notarztbesetzten Rettungswesen wie in Deutschland beginnt die Therapie Schwerverletzter bereits in der Präklinik. Hier werden wegweisende und ergebnisentscheidende Weichen gestellt. Vieles bleibt jedoch noch ungeklärt und so kann das Ziel der präklinischen Versorgung nur die initiale lebensrettende Stabilisierung des Patienten sein, um ihn möglichst rasch in das geeignete Traumazentrum zu transportieren. Kaum ein Arbeitsumfeld wird derart umfangreich mit Kursformaten (Advanced Trauma Life Support [ATLS ${ }^{\circledR}$ ], European Trauma Course $\left[\mathrm{ETC}^{\circledR}\right]$, High Fidelity Simulationstraining für mehr Sicherheit im Schockraum [HOTT ${ }^{\circledR}$ ] etc.) abgedeckt und beübt wie die Schnittstelle Schockraum. Diese Notwendigkeit liegt darin begründet, dass ein Patient zu versorgen ist, dessen Gesamtsituation zum Zeitpunkt der Einlieferung noch unklar ist und jederzeit eine pathologische Dynamik aufweisen kann. Zum anderen wird für das interdisziplinäre und interprofessionelle Team, das sich erst kurz vor Ankunft des Patienten im Schockraum zusammenfindet, vorausgesetzt, dass umfangreiche interpersonelle Kompetenzen bestehen. Die Kernkompetenzen eines Schockraumleaders müssen neben der medizinischen Kompetenz nach ATLS-Richtlinien vor allem auf dem Gebiet des Zeit- und Personalmanagements liegen.

Die S3-Leitlinie Polytrauma/Schwerverletzten-Behandlung widmet dem Schockraummanagement ein umfangreiches Kapitel und konstatiert neben Voraussetzungen für die Ausstattung eines Schockraums auch das Management von der Diagnostik bis zur interdisziplinären Entscheidungsfindung. Feste Schockraumteams sollten nach vorstrukturierten Plänen arbeiten und/oder ein spezielles Training absolviert haben. Der Anspruch ist es demnach, im Schockraum das Verletzungsmuster des Patienten vollumfänglich zu erfassen, gleichzeitig aber keine Zeit zu verlieren, um die für das Schockraummanagement mobilisierten Ressourcen nicht allzu lange zu binden. Es gilt, den Patienten optimal zu therapieren und Entscheidungen unter interdisziplinärer Priorisierung für das weitere Vorgehen rasch herbeizuführen. Während die optimale Schockraumversorgung das Ziel aller ist, muss gleichzeitig auch die Versorgung anderer Patienten weiterlaufen und so sollte die Klinik in ihrem Alltagsablauf dadurch nicht wesentlich unterbrochen werden.

An der Versorgung eines Polytraumas sind in den ersten $24 \mathrm{~h}$ bis zu 120 Personen beteiligt. Daher werden vermehrt Forderungen nach abgestuften Schockraumalarmierungen laut, die es erlauben, zum einen an den Patienten angepasste individualisierte und gleichzeitig definierte Versorgungsstandards abzurufen und zum anderen Ressourcen bedarfsanagepasst zu mobilisieren. Dadurch sollen Übertriagierungen reduziert und Untertriagierungen vermieden werden.

In der vorliegenden Darstellung soll anhand des Göttinger Modells ein etabliertes bedarfs- und ressourcenangepasstes Schockraumalarmierungssystem und -management dargestellt werden. Die Etablierung und umfangreiche Auseinandersetzung mit diesem System ist in [1] dargestellt. Das Göttinger Schockraumalarmierungsschema mit einem 3- bzw. 5-stufigen Modell (Grün, Gelb, Rot sowie Blau und Grau) stellt ein fest etabliertes bewährtes System zur bedarfsgerechten interdisziplinären Schockraummobilisation dar. Das Weißbuch Schwerverletztenversorgung der $D G U^{\circledR}$ widmet sich in der 3 . Auflage ebenfalls diesem Thema, stellt aber auch deutlich heraus, dass eine Abstufung eher im Sinne eines Eskalationspotenzials von einem Basisteam ausgehend zu verstehen ist und die stufenweise Schockraumalarmierung nur in den größeren Traumazentren sicher praktiziert werden kann. Die interdisziplinäre Entscheidungskompetenz zur Diagnostik und operativen Priorisierung sollte im Schockraum vertreten sein, um nicht wertvolle Zeit verstreichen zu lassen.

In einer prospektiven Datenerhebung wurden sowohl die Schockraumalarmierung als auch das Zeitmanagement während der Schockraumversorgung nach Einführung des abgestuften Schockraumalarmierungssystems reevaluiert. Es zeigt sich seit Einführung eines strukturierten Alarmierungssystems für den Schockraum eine Zunahme an Schockraumaufnahmen bei nahezu gleichbleibender durchschnittlicher Krankenhausliegedauer, leicht sinkender Letalität und leicht steigender Verletzungsschwere. Gleichzeitig findet sich eine stabil niedrige Rate der Überund Untertriage sowie eine stabile Korrelation der Anmel- 


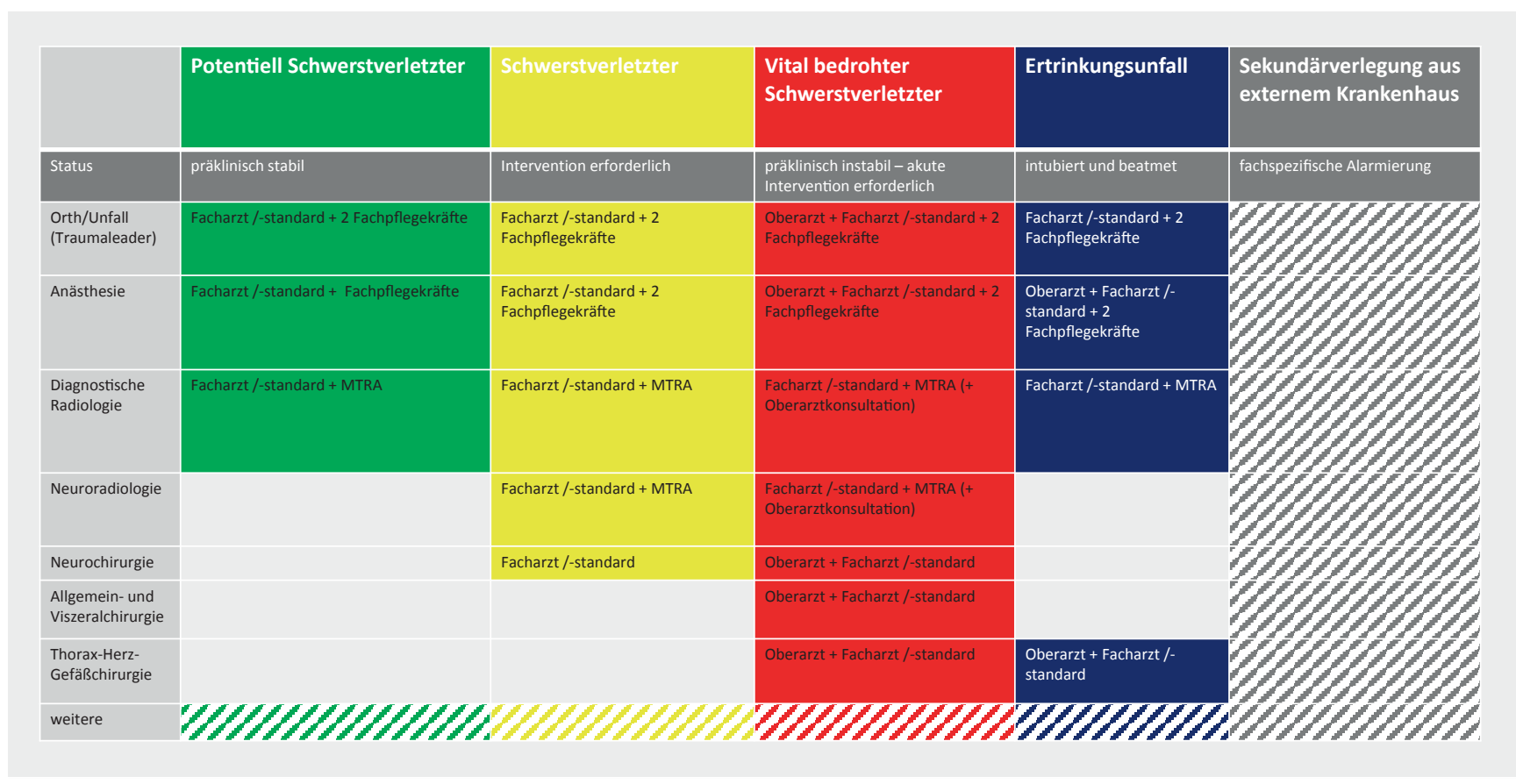

- Abb. 1 Ressourcenoptimiertes und an den Zustand des Patienten angepasstes Schockraumalarmierungsschema der Klinik für Unfallchirurgie, Orthopädie und Plastische Chirurgie der Universitätsmedizin Göttingen. Die Anzahl der Teammitglieder bezieht sich auf ärztliches (Facharzt oder Facharztstandard) und pflegerisches Personal bzw. medizinisch-radiologisch-technische Assistenz (MRTA).

destufe mit der später erhobenen Verletzungsschwere (Injury Severity Score [ISS] in Punkten).

Die Daten zeigen damit, dass ein strukturiertes Schockraumalarmierungssystem in definierten Stufen und klarer Kommunikation an die präklinischen Rettungsteams, inkl. Schulungen in der Anmeldesystematik, nicht nur die Ressourcenmobilisation effizienter gestaltet, sondern auch zu einer sichereren Patientenversorgung führen kann.

\section{Schockraumalarmierung und Triage}

Das bereits etablierte und bekannte 3-stufige Schockraumalarmierungsschema mit

1. Grün: potenziell Schwerstverletzter - präklinisch stabil,

2. Gelb: Schwerstverletzter - präklinisch stabilisierbar Intervention erforderlich und

3. Rot: vital bedrohter Schwerstverletzter - präklinisch instabil - akute Intervention erforderlich

wurde bereits frühzeitig ergänzt durch die Alarmierungsstufen:

4. Blau: Ertrinkungsunfall - intubiert und beatmet und

5. Grau: Sekundärverlegung aus externem Krankenhaus (vgl. > Abb. 1).

Die Schockraumalarmierung wird damit vollständig vom in der Präklinik den Patienten behandelnden notärztlichen Rettungsteam indiziert und triagiert. Hierbei wird der von Spering et al. veröffentlichte Algorithmus verwendet ( $\mathrm{vgl}$ > Abb.2), der die A- und B-Kriterien zur Schockraumalarmierung der S3-Leitlinie Polytrauma/ Schwerverletzten-Behandlung der DGU beinhaltet.

Die Grundlage des Erfolgs dieses Schemas ist die klare Kommunikation vom Unfallort in die Klinik und das Vertrauen in die Fertigkeit der richtigen präklinischen Triage. Bei der Anmeldung eines Schockraumpatienten an der klinikinternen Leitstelle werden daher nur klar kommunizierte und auf das Wesentliche reduzierte Informationen ausgetauscht:

1. Anzahl der Patienten

2. Schockraumalarmierungsstufe

3. Ankunftsuhrzeit

4. Verletzungsmechanismus

5. hämodynamische Stabilität

6. Atemwegssicherung

Von der Leitstelle aus wird umgehend die angeforderte Teamzusammensetzung nach dem Schema in $\mathbf{A}$ Abb. 1 alarmiert. Sollten weitere Informationen notwendigerweise ausgetauscht werden, können diese während der Anfahrt über das Traumahandy an den diensthabenden Schockraumleader (Unfallchirurg) direkt per Arzt-ArztKommunikation übermittelt werden.

Das Basisteam bestehend aus Unfallchirurg, Anästhesist, diagnostischem Radiologen, Fachbereichspflege Unfallchirurgie, Fachpflege Anästhesie und medizinisch-tech- 


\begin{tabular}{|c|c|c|}
\hline Zustand des Patienten & SR-Aufnahmekriterien & Alarmierungsstufe \\
\hline Stabiler Patient nach Trauma & Keine Anhaltspunkte für ein Hochrasanztrauma & Chirurgische Notaufnahme \\
\hline $\begin{array}{l}\text { Stabiler Patient nach Hochrasanztrauma } \\
\text { oder mit schwerer Monoverletzung }\end{array}$ & $\begin{array}{l}\text { Unfallmechanismus } z . B .: \\
\text { - Sturz }>3 \text { m Höhe } \\
\text { - Verkehrsunfall mit: }>30 \mathrm{~km} / \mathrm{h} \text { Beschleunigungsdifferenz, Tod eines Insassen, Ejektion } \\
\text { aus Fahrzeug, hohe Verformung des Fahrzeugs } \\
\text { - Motorradunfall mit }>30 \mathrm{~km} / \mathrm{h} \text { Aufprallgeschwindigkeit } \\
\text { - PKW vs. Fußgänger / Zweiradfahre }\end{array}$ & $\begin{array}{l}\text { Schockraumstufe grün } \\
\text { potenziell Schwerstverletzter }\end{array}$ \\
\hline $\begin{array}{l}\text { Stabiler Patient mit aus adäquatem } \\
\text { Unfallmechanismus folgendem } \\
\text { Verletzungsmuster und/oder } \\
\text { traumaassoziierten Symptomen }\end{array}$ & $\begin{array}{l}\text { Verletzungsmuster z.B.: } \\
\text { - } \quad \text { zwei oder mehr Körperregionen verletzt } \\
\text { Traumaassoziierte Symptome z.B.: } \\
\text { - Blutdruck systolisch }<90 \mathrm{mmHg} \\
\text { - } \quad \text { GCS }<14 / 15 \\
\text { - Atemfrequenz }<10 / \text { min oder }>29 / \mathrm{min}\end{array}$ & \\
\hline $\begin{array}{l}\text { Instabiler Patient nach adäquatem } \\
\text { Unfallmechanismus, Verletzungsmuster } \\
\text { und / oder negativer Dynamik, präklinisch } \\
\text { nur unzureichend stabilisierbar }\end{array}$ & $\begin{array}{l}\text { Instabilität } z . B \text {. } \\
\text { - unter präklinischen Maßnahmen stabilisiert sich der hämodynamische und/oder } \\
\text { respiratorische Zustand nicht oder unzureichend. } \\
\text { - zerebrale Einklemmungssymptomatik } \\
\text { - negative Dynamik eines initial niedriger eingestuften Patienten während des } \\
\text { Transports }\end{array}$ & $\begin{array}{l}\text { Schockraumstufe rot } \\
\text { Vital bedrohter Schwerstverletzter }\end{array}$ \\
\hline $\begin{array}{l}\text { Ertrinkungsunfall mit Indikation zur } \\
\text { Intubation und Beatmung }\end{array}$ & $\begin{array}{l}\text { Intubiert, beatmeter Patient mit z.B. } \\
\text { - } \quad \text { erniedrigter Körperkerntemperatur } \\
\text { - } \quad \text { Aspiration }\end{array}$ & Schockraumstufe blau \\
\hline $\begin{array}{l}\text { In einem anderen Traumazentrum bereits } \\
\text { intial stabilisierter und/oder therapierter } \\
\text { Patient }\end{array}$ & $\begin{array}{l}\text { Sekundärverlegung aufgrund z.B. } \\
\text { - } \quad \text { spezielle fachspezifische Therapie } \\
\text { - } \quad \text { komplexes Polytrauma nach initialer Stabilisierung }\end{array}$ & $\begin{array}{l}\text { Schockraumstufe grau } \\
\text { Fachspezifische Alarmierung }\end{array}$ \\
\hline
\end{tabular}

- Abb. 2 Präklinische Vorgehensweise zur Triage und Ressourcenmobilisation in der Zielklinik.

nischer Assistenz Radiologie (MRTA) wird bei jeder Schockraumalarmierung mobilisiert. Je nach Anmeldestufe werden weitere Fachdisziplinen hinzugezogen (vgl. - Abb. 2a). In dem Sonderfall „Ertrinkungsunfall“ mit einem intubierten und beatmeten Patienten werden frühzeitig zusätzlich zu dem Basisteam der Kardiotechniker und der Diensthabende der Thorax-Herz-Gefäßchirurgie sowie der Oberarzt alarmiert. Bei der grauen Schockraumalarmierung handelt es sich um eine fachspezifische Sekundärverlegung aus einem externen Krankenhaus. Hier wird das Team durch die den Patienten annehmende Fachdisziplin zusammengestellt und entsprechend über die Leitstelle der Klinik alarmiert (vgl. - Abb. 2 b).

Die Evaluation der Verlässlichkeit der präklinisch eingestuften Patienten zeigt, dass in den meisten Fällen die durch den Notarzt angemeldete Schockraumalarmierungsstufe mit der später ermittelten Verletzungsschwere korreliert. Dies ist weitgehend über die Jahre konstant (vgl. > Abb. 4). Mit zunehmender Routine in dem praktizierten Alarmierungssystem verbessert sich deren Qualität, gemessen an der Über- und Untertriage. Während im Zeitraum 2015/16 die Übertriage bei 28\% und die Untertriage bei $7 \%$ lagen, zeigt sich im Zeitraum 2017/18 eine Übertriage von $22 \%$ und eine Untertriage von $6 \%$ bei gleichzeitig steigender Anzahl an Schockraumalarmierungen (2015: 769, 2018: 871; vgl. > Abb. 6).
Der kritischen Auseinandersetzung mit der Notwendigkeit einer notärztlichen Versorgung bzw. Indikationsstellung zur Schockraumalarmierung ist mit diesen Daten und dem hier praktizierten Ressourcenmobilisationssystem eine klare Notwendigkeit entgegenzubringen. Auch wenn aktuelle Auswertungen aus dem TraumaRegister DGU die notärztliche Präsenz bei der Versorgung von Schwerverletzten in der Präklinik im Allgemeinen kritisch hinterfragen, sehen wir bei der aufgezeigten Verkettung von Indikation und Ressourcenmobilisation sowie Stabilisierung und Vorbereitung des Patienten die notärztliche Kompetenz in der Präklinik bis auf wenige Ausnahmen als unerlässlich an.

\section{Vorbereitung des Schockraums}

Die Alarmierung des Teams erfolgt über die klinikinterne Leitstelle. Hierbei werden nur die o.g. 6 Informationen weitergeleitet. Diskussionen oder Rückfragen erfolgen hier nicht. Das gesamte Team soll den Patienten vollständig $24 \mathrm{~h}$ am Tag bei dessen Ankunft im Schockraum erwarten. Dies gelingt nahezu immer $(99,4 \%)$ ab einer Vorlaufzeit von 6 min unabhängig von der Alarmierungsstufe. Bei einer Vorlaufzeit von unter 3 min gelingt dies in noch 54-78\% der Fälle, abhängig von der Alarmierungsstufe (vgl. > Abb. 3a, b). Die oberärztliche Präsenz außerhalb der Regelzeit bei Alarmierungsstufe Rot sollte innerhalb von 20 min ab Alarmierung gegeben sein. Je nach 


Stufe Grün - Potentiell Schwerverletzter, präklinisch stabil
Auf Grund des Unfallhergangs / der Unfallikinetik sofortige Diagnostik und Uberwachung erforderlich
Präsenz: FA. -standard + Fachbereichspflege Unfallichiurgie
FA,-standard + Fachplege Anästhesiologie
FA -standard + MTAR Diagnostische Radiologie
Auf Anforderung weitere Disziplinen

\section{Stufe Gelb - Schwerverletzter, Intervention erforderlich}

Pat. mit präklinisch dringendem VerdachtAnzeichen auf schwere Verletzungen wie z.B. SHT mit Bewusstseinseintrübung. Thoraxtrauma mit V.a. Pneumothorax bei resp. Insuffizienz, stumpfes Bauchtrauma mit V.a. Organverletzung bei hämodynamischen Auffälligkeiten, Extremitätenverletzung bei offener oder geschlossener Fehlstellung.

Präsenz: $\quad$ FA, -standard + Assistent +2 Fachbereichspfleger Unfallchirurgie

FA, -standard + Assistent + Fachpfleger Anästhesie

FA, -standard + jeweils MTAR Diagn. Radiologie / Neuroradiol.

FA, -standard Neurochirurgie

Auf Anforderung: FA, -standard Allgemeinchirurgie und/oder THG und weitere Fachdisziplinen

Logistik: Radiologie: Bereitschaft für sofortiges Röntgen + Traumaspirale
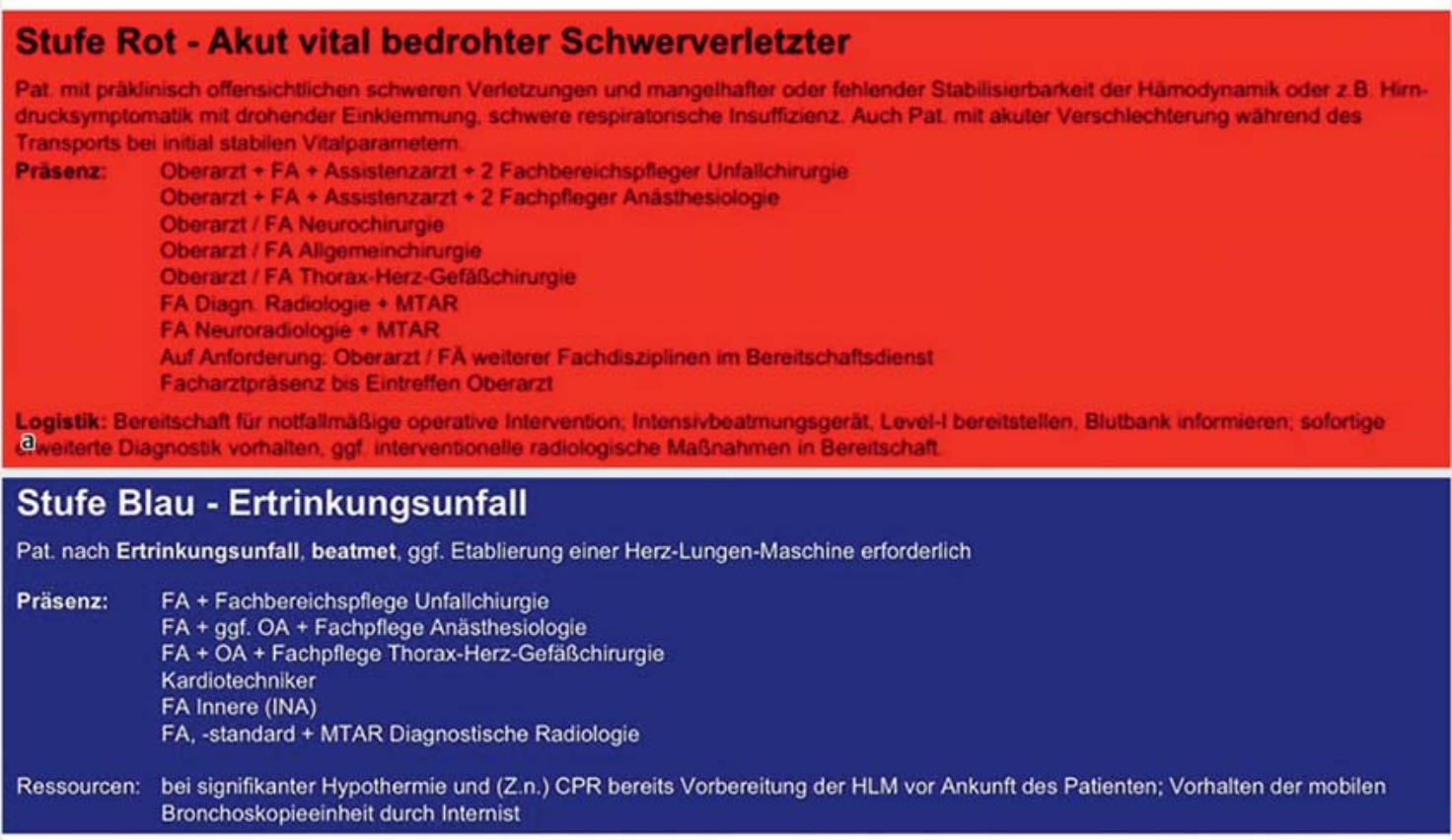

\section{Stufe Grau - Fachspezifische Verlegung aus ext. Krankenhaus}

Fachspezifische Sekundärverlegung aus externem Krankenhaus z.B. Patient mit isoliertem akutem Subduralhämatom für die Abtlg. Neurochirurgie oder isolierte Milzruptur für die Abtlg. Allgemeinchirurgie. Die initiale Diagnostik ist bereits durch die Primärklinik erfolgt. Verlegung über fachspezifischen Arzt-Arzt-Kontakt zwischen Primärklinik und jeweiligem AvD.

Die Absprache mit dem LKE und der chirurgischen Notaufnahme sowie dem 1. Dienst Unfallchirurgie ist aufgrund der Schockraumbelegung obligat und obliegt der den Patienten annehmenden Fachdisziplin.

Konsiliarische Mitbehandlung durch andere Disziplinen erfolgt nur nach direkter Aufforderung durch den AvD der den Patienten annehmenden Fachdisziplin.

Präsenz: FA, -standard + Fachbereichspfleger der annehmenden Fachdisziplin. Die Präsenz weiterer Disziplinen erfolgt nur nach Aufforderung burch den AvD der den Patienten annehmenden Fachdisziplin.

- Abb. 3 Ressourcenmobilisation je nach Anmeldestufe mit entsprechender personeller Ausstattung. Quelle: Spering C, Roessler M, Kurlemann T et al. Optimierte Ressourcenmobilisation und Versorgungsqualität Schwerstverletzter durch eine strukturierte Schockraumalarmierung. Unfallchirurg 2018; 121: 893-900. Neu hinzugekommen ist die blaue Stufe. [rerif]

Alarmierungsstufe werden neben dem vollständigen Team auch Ressourcen vorbereitet. Dies ist zum einen das Röntgen und das CT, bei roten Schockraumalarmierungen (z.B. Massentransfusionseinrichtungen; Level I) sowie speziellen Maßnahmen (z. B. Thoraxdrainage oder spezielle OP-Siebe) je nach Anmeldekonstellation und Zusatzinformation. Die jeweiligen Teammitglieder sind durch farblich gekennzeichnete und mit Aufschrift versehene Röntgenschürzen für das Team selbst und für externe erkennbar (vgl. > Abb. 5). 


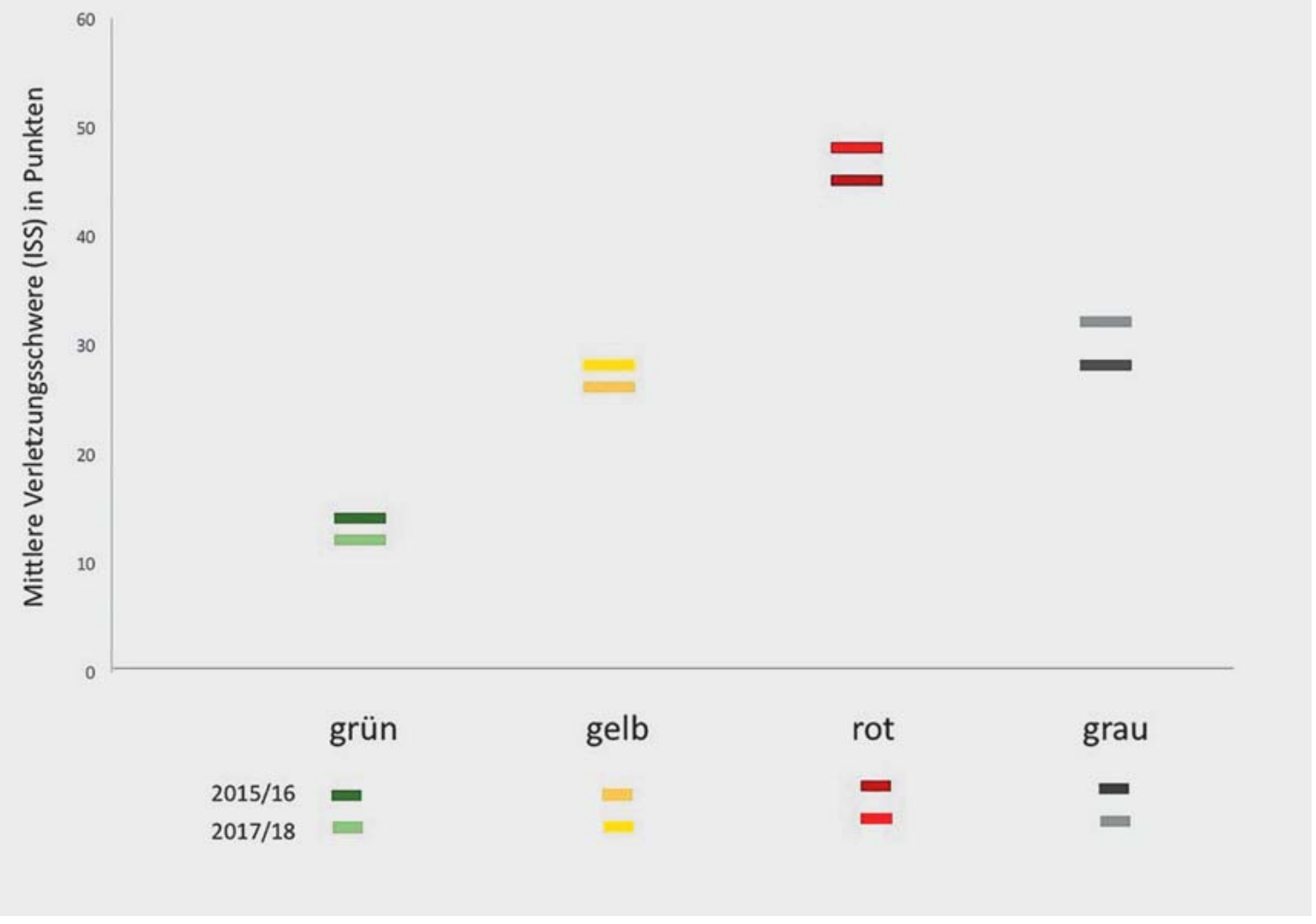

- Abb. 4 Korrelation von Verletzungsschwere (mittlerer Injury Severity Score - ISS: 0-75 Punkte) und der angemeldeten Schockraumalarmierungsstufe.

Der Schockraum an sich ist baulich so eingerichtet, dass entlang des Patienten die Versorgungsschränke mit A, B, $C$, D und $E$ gekennzeichnet sind, wobei A und B in Höhe des Kopfes des Patienten und D/E in Höhe des Fußendes eingerichtet sind. Der Schockraum selbst sollte laut Angaben aus dem Weißbuch Schwerstverletztenversorgung DGU sowie der S3-Leitlinie Polytrauma/Schwerverletzten-Behandlung mindestens $25-50 \mathrm{~m}^{2}$ groß sein und entweder ein CT als „Sliding Gentry“ enthalten oder eines in unmittelbarer Nähe und auf gleicher Etage vorhalten. Bei dem Betreiben von mehreren Schockräumen gleichzeitig zur simultanen Versorgung mehrerer Patienten sollten die Kommunikations- und Laufwege zwischen den Schockräumen möglichst kurz und barrierefrei sein, um spezielle Fachkompetenz auszutauschen und klare Absprachen treffen zu können. Der Weg in den OP und auf die Intensivstation sollte kurz sein, die Kommunikation in den OP hinein zur Vorbereitung einer folgenden operativen Versorgung oder auf die Intensivstation zur weiteren Stabilisierung sollte ebenfalls kurz sein und frühzeitig erfolgen.

\section{Schockraummanagement}

Die Versorgung (potenziell) Schwerverletzter stellt das Schockraumteam aufgrund der Komplexität der jeweiligen Prozesse und Zusammenhänge vor eine große Herausforderung. Grundlage ist das Verständnis und die Übersicht über die abgelaufenen Prozesse vor der Schockraumzuweisung sowie die Entscheidungsfindung und Vorgehensweise für die Weiterbehandlung. Das Schockraummanagement stellt somit das wichtigste Bindeglied dar. Während dieser wichtigen Phase werden entscheidende Weichen gestellt, da die Schockraumversorgung hohe Relevanz für das Überleben des Patienten hat. Grundlage für das erfolgreiche Management der komplexen Abläufe und medizinischen Zusammenhänge ist die klare und zielgerichtete Kommunikation sowie das Beherrschen von Algorithmen. Um dies zu gewährleisten, existieren Kurskonzepte, wie Pre-Hospital Trauma Life Support $\left(\mathrm{PHTLS}^{\circledR}\right)$ für die präklinische Versorgung und ATLS oder ETC für die klinische Versorgung. Sie können durch eine Priorisierung der Behandlungsabläufe und eine gemeinsame Sprache Prozesse automatisieren und verbessern. Mit Ankunft des Rettungsdienstteams mit dem Patienten im Schockraum wird eine für alle sichtbare 


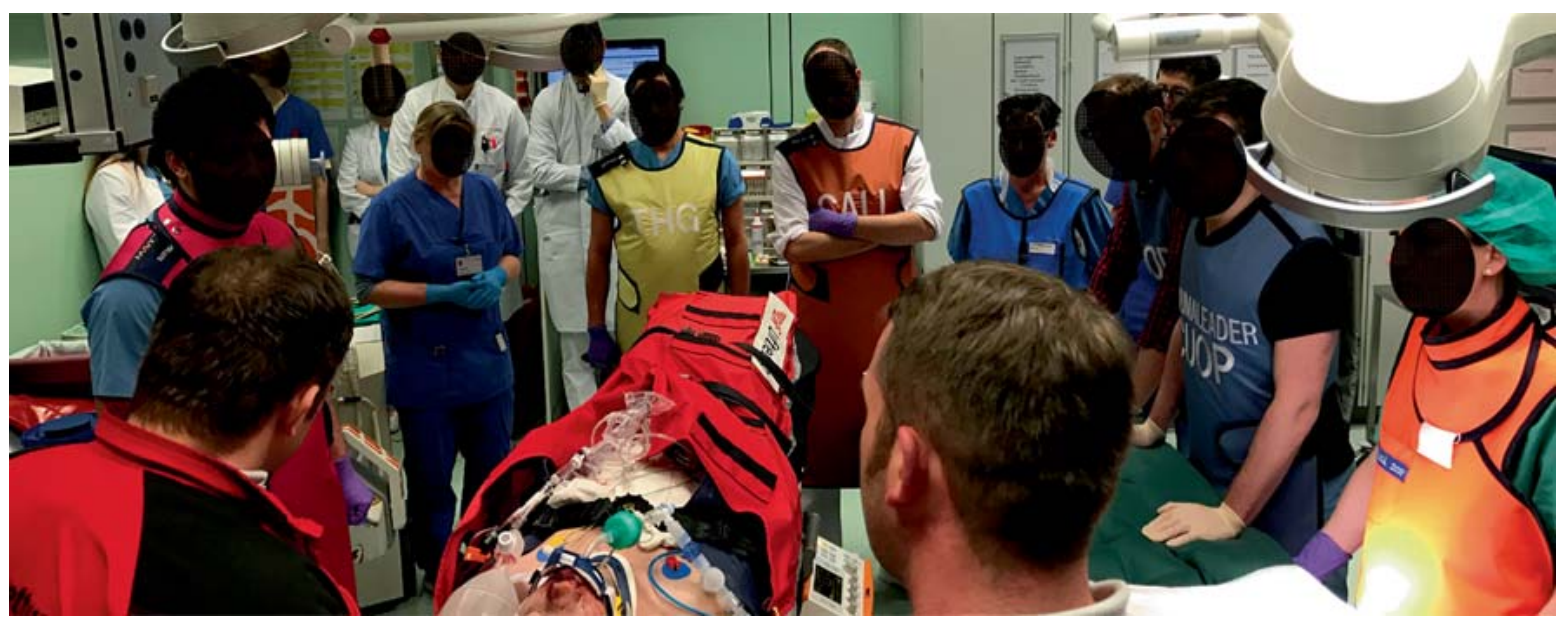

- Abb. 5 Interdisziplinäres Schockraummanagement eines als „rot“ eingelieferten Motorradfahrers. Farblich und textlich gekennzeichnete Fachvertreter von CUOP: Unfallchirurgie, Traumaleader, NCHI: Neurochirurgie, CALL: Allgemeinchirurige, THG: ThoraxHerz-Gefäßchirurgie, ANAE: Anästhesie und Radiologie.

Stoppuhr gestartet. Das Schockraummanagement beginnt mit einer sog. „5-Sekunden-Visite“ durch den Traumaleader. Hierbei wird mindestens per Blickdiagnose der allg. Zustand des Patienten erfasst inkl. Beatmungssituation und ggf. bestehender aktiver Blutung. Dieser kurze Überblick ist notwendig, um für etwaige unmittelbar lebensbedrohliche Zustände nicht wichtige Zeit zu verlieren. Die folgende Übergabe vom Notarzt an das Schockraumteam sollte dabei den o.g. Algorithmen und der gemeinsamen Sprache folgen, trotz einer z. B. laufenden mechanischen kardiopulmonalen Reanimation.

Während der Übergabe ist alle Aufmerksamkeit auf den Notarzt gerichtet (vgl. > Abb. 5), damit wesentliche Informationen nicht unnötig wiederholt werden müssen. Damit ggf. gezielt Fachvertreter mit spezifischen Informationen auch vom Rettungsdienstteam angesprochen werden können, sollten die jeweiligen Teammitglieder durch farblich gekennzeichnete und/oder beschriftete Schürzen (in diesem Fall Röntgenschürzen) kenntlich sein (vgl. > Abb. 5). Diese Teams sollten - wenn auch die jeweilige Zusammenstellung jedes Mal variieren kann - regelmäßige Schockraumtrainings absolvieren und ihre Arbeit in einem regelmäßig stattfindenden innerklinischen Qualitätszirkel evaluieren. Das Basis-Schockraum-Team Grün im vorliegenden Schockraummodell besteht aus Unfallchirurgie, Anästhesie, Diagnostischer Radiologie, Fachbereichspflege Unfallchirurgie, Fachpflege Anästhesie und MRTA. Mit diesem Team kann das Schockraummanagement im Normalfall bis zur Durchführung des Ganzkörper-CTs ablaufen. Bei spezifischen Herausforderungen oder Fragestellungen kann es jederzeit erweitert werden. Bis zum Durchführen des eFAST (extended focused assessment with sonography in trauma) sollten ab Ankunft des Patienten im Schockraum nicht mehr als 3 min vergehen, bis zum Durchführen des Ganzkörper-
CTs nicht mehr als 10 min, sofern der Zustand des Patienten dies erlaubt.

Der Schockraumleader oder die „interdisziplinäre Führungsgruppe“ leiten das Team, das parallel A-, B-, C- und D-Probleme ausschließt oder abarbeitet. Voraussetzung dafür ist der stets bestehende Austausch von Befunden während der Befunderhebung sowie für alle sichtbare Monitore mit Vitalparametern und ggf. Bildgebung. Dies kann bspw. durch Großbildschirme, die für alle sichtbar Überwachungsmonitore spiegeln, gelöst sein. Für spezifische medizinische Herausforderungen sollten Standard Operating Procedures (SOPs) standortspezifisch definiert sein. Ferner sollten die Abläufe und Zuständigkeitsbereiche im Schockraum durch einen Algorithmus klar definiert zugewiesen sein. Diese Abläufe und „Spielregeln“ sollten nicht nur klar kommuniziert, sondern vor allem auch trainiert werden. Für die Durchführung von notfalloperativen Eingriffen im Schockraum - ggf. sogar vor der Bildgebung - sollten entsprechende Ressourcen wie OPSiebe u.a. im Schockraum vorgehalten werden. Eine Eskalation in Richtung notfallmäßige Damage Control sollte stets als Option möglich sein und sowohl vonseiten der Ressourcen als auch des Personals vorgehalten werden.

Für kleinere Traumazentren mit weniger interdisziplinären Ressourcen sollte die lebenswichtige Stabilisierung des Patienten im Vordergrund stehen und eine ggf. frühzeitige Sekundärverlegung in ein Zentrum einer höheren Versorgungsstufe initiiert werden. Nicht zuletzt die strukturierte und durch einen interdisziplinären Qualitätszirkel stets evaluierte Versorgungsqualität führen dazu, dass trotz steigender Zahl der Aufnahmen über den Schockraum und gleichzeitig steigender mittlerer Verletzungsschwere (gemessen als ISS) sowohl die Krankenhausletalität als auch die Verweildauer nicht nur konstant bleiben, 


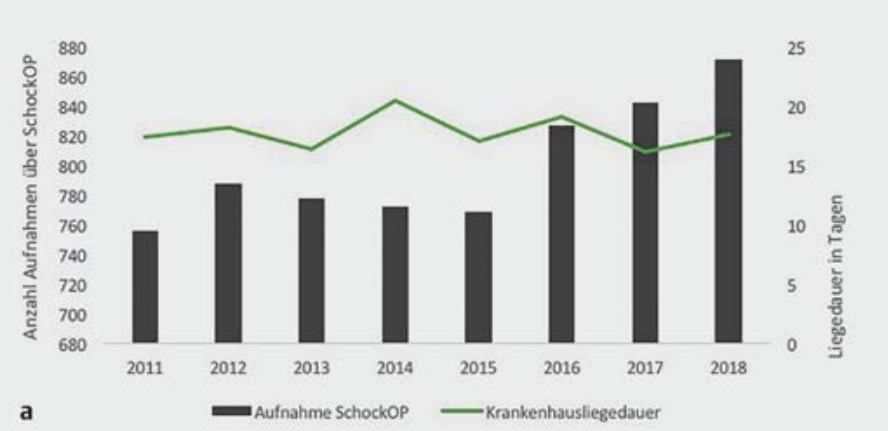

a Aufnahme SchockOP Krankenhausliegedauer

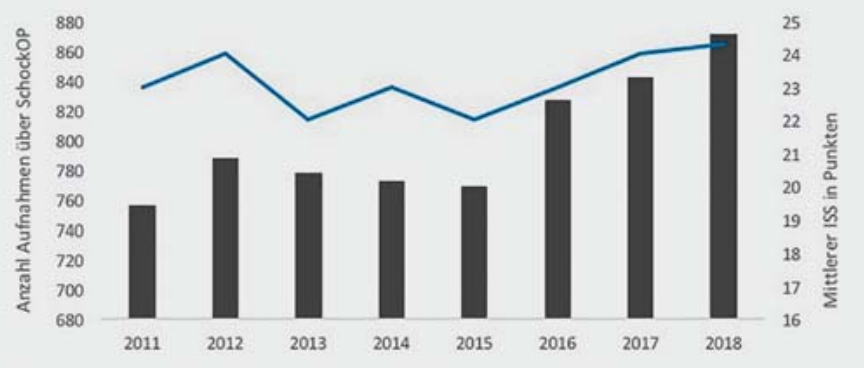

b
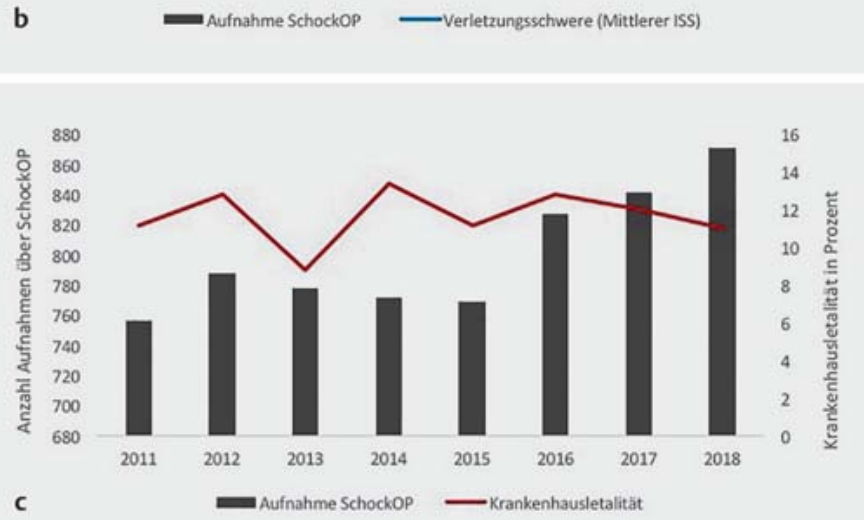

c

Aufnahme Schockop Krankenhausletalitat

- Abb. 6 Korrelation von Aufnahmeanzahl über den Schockraum und (a) Krankenhausliegedauer, (b) Verletzungsschwere und (c) Krankenhausletalität.

\section{Besondere Situationen}

\section{Pädiatrische Schwerverletzte}

Das Management von Schwerverletzten ist insbesondere bei Patienten unter 14 Jahren aus 2 Gründen eine besondere Herausforderung für das Team:

1. Es besteht eine Unsicherheit in Dosierungen und Algorithmen, da pädiatrische Polytraumen (ca. 7\% der Schwerverletzten) selten sind und

2. es weckt eine eigene Emotionalität bei den Teammitgliedern.

Daher wird empfohlen, das Schockraumteam um den pädiatrischen Intensivmediziner zu erweitern und den Allgemeinchirurgen durch den Kinderchirurgen zu ersetzen. Fakultativ sollte - wenn vorhanden - auch der Kinderneurochirurg bereits in den Schockraum gerufen werden. Ferner sollten frühzeitig ein Kinderintensivbett und eine kinderanästhesiologische Kompetenz abgerufen werden.

Im Schockraummanagement ist es essenziell, dass bereits vor Ankunft des Patienten die Oberärzte vor Ort sind, da bereits die Entscheidung über CT-Bildgebung oder operative Indikationsstellungen frühzeitig und interdisziplinär kommuniziert werden sollten. Die eFAST-Diagnostik sollte stets vorgezogen werden. Jedoch muss auch die Ganzkörper-CT-Untersuchung bei Unklarheit oder hoher kinetischer Energie im Team gemeinsam mit den Pädiatern und Radiologen besprochen und bei Zweifel eher großzügig indiziert werden. Dabei ist zu beachten, dass neuroradiologische CT-Befundungen in ihrer Diagnostik oft unsicher sind, da die Hirnmasse und die CT-morphologische Darstellung anders als bei Erwachsenen sind. So sind Kontusionen, Ödeme und kleine Blutungen nicht immer sicher abgrenzbar.

Während der gesamten Versorgung im Schockraum und im CT ist auf ausreichende Wärmezufuhr, möglichst über eine Wärmematte und entsprechende Temperaturanpassung der Raumluftsteuerung zu achten. Die Thermoregulation ist einer der ersten Parameter, der bei traumatisierten Kindern verändert ist und den Zustand des Kindes wesentlich negativ beeinflussen kann. Es empfiehlt sich, einen gesonderten vollständig ausgestatteten Versorgungswagen für Kinder bereitzuhalten, in dem pädiatrische Größen für Intubationsbesteck, Tuben, Zugänge, ggf. Thoraxdrainagen etc. vorgehalten werden. Unterstützend für alters- und vor allem gewichtsgerechte Dosierungen kann das Breslow ${ }^{\circledR}$ Pediatric Emergency Tape zur Hilfe genommen werden ( $\bullet$ Abb. 7). Bei der Lagerung und dem Management sind einige Besonderheiten zu beachten:

- Je kleiner das Kind desto größer das disproportionale Verhältnis zwischen Schädel und Mittelgesicht $\rightarrow$ Kinder zwischen 1 und 3 Jahren mit dem Rumpf auf 3 cm dickes Polster lagern („Schnüffelposition“). 

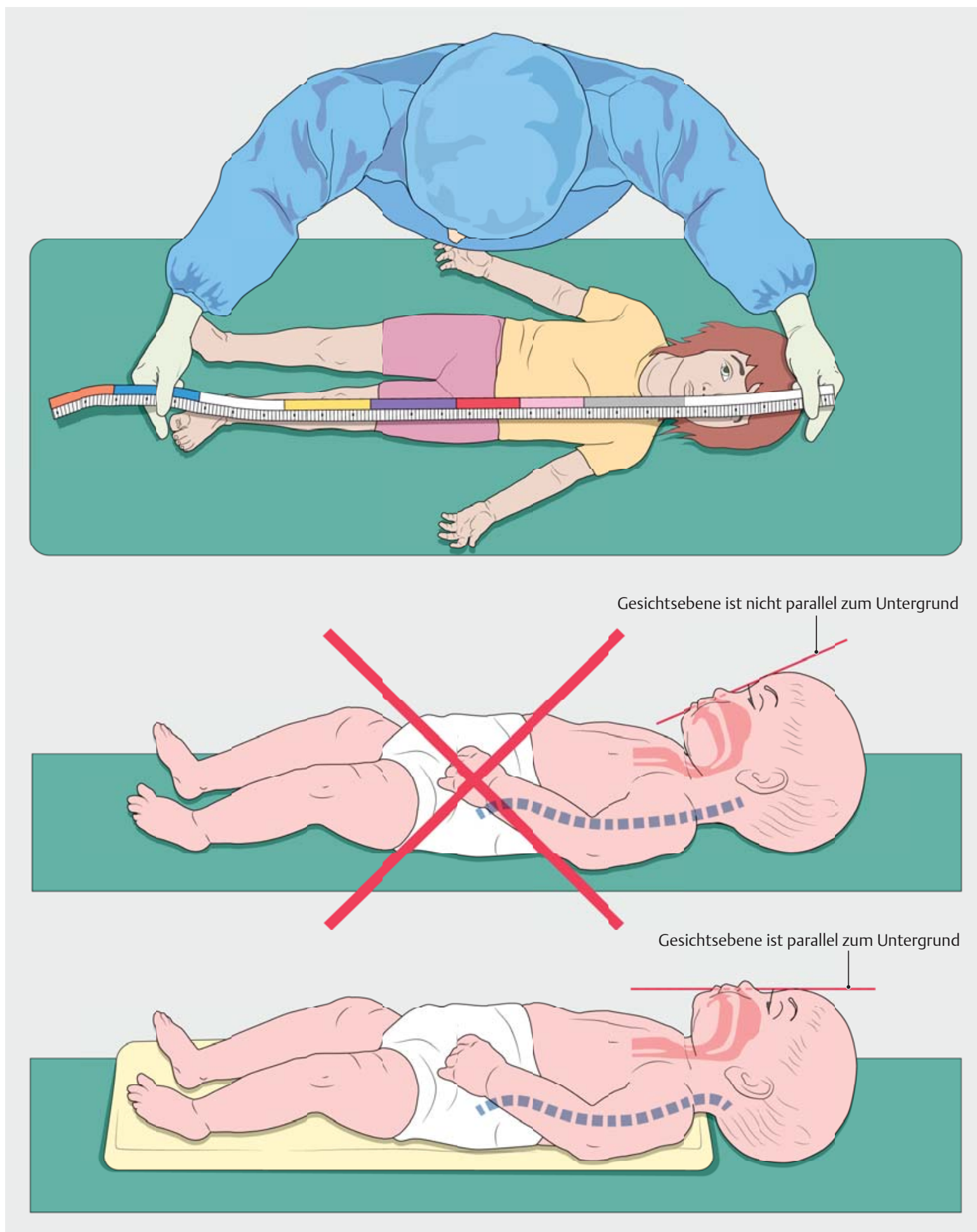

- Abb. 7 Breslow ${ }^{\circledR}$ Pediatric Emergency Tape; Schnüffelposition eines Säuglings auf der Schockraumtrage.

- Atemnot von Ateminsuffizienz unterscheiden $\rightarrow$ Ziel ist eine gute Oxygenierung, insbesondere im Trauma.

- Auch bei Säuglingen/Kindern ist eine chirurgische Koniotomie als alternative Atemwegssicherung möglich, aber das Lig. cricothyroideum ist erst ab dem 12. LJ gut palpierbar.
- Das Atemzugvolumen (AZV) 4-6 ml/kg, bei Maskenbeatmung ist das AZV meist höher: Achtung iatrogenes Barotrauma!

- Hypoxie ist die häufigste Ursache für Herzstillstand bei Kindern. Zuvor ist oft Hypoventilation Ursache der Hypoxie mit folgender Azidose (häufig bei schwer ver- 
- Tab. 1 Tabelle zu Normwerten bei Kindern, altersentsprechend sortiert.

\begin{tabular}{|c|c|c|c|c|c|}
\hline & $\begin{array}{l}\text { Gewicht } \\
\text { [kg] }\end{array}$ & $\begin{array}{l}\text { Herzfrequenz } \\
\text { [pro min] }\end{array}$ & $\begin{array}{l}\text { Blutdruck } \\
\text { [mmHg] }\end{array}$ & $\begin{array}{l}\text { Atemfrequenz } \\
\text { [pro min] }\end{array}$ & $\begin{array}{l}\text { Urinausscheidung } \\
{[\mathrm{ml} / \mathrm{kg} / \mathrm{h}]}\end{array}$ \\
\hline Säugling, 0-12 Monate & $0-10$ & $<160$ & $>60$ & $<60$ & 2,0 \\
\hline Kleinkind, 1-2 Jahre & $10-14$ & $<150$ & $>70$ & $<40$ & 1,5 \\
\hline Vorschulkind, 3-5 Jahre & $14-18$ & $<140$ & $>75$ & $<35$ & 1,0 \\
\hline Schulkind, 6-12 Jahre & $18-36$ & $<120$ & $>80$ & $<30$ & 1,0 \\
\hline Jugendliche(r), > 13 Jahre & $36-70$ & $<100$ & $>90$ & $<20$ & 0,5 \\
\hline
\end{tabular}

- Tab. 2 Tabelle zur Orientierung bei Blutverlust von Kindern und den zu erwartenden Kompensationsmechanismen bei entsprechenden Mengen.

\begin{tabular}{|l|l|l|l|}
\hline System & leichter Blutverlust $\mathbf{3 0 \%}$ & mittlerer Blutverlust $\mathbf{3 0 - 4 5 \%}$ & schwerer Blutverlust $\mathbf{4 5 \%}$ \\
\hline kardiovaskulär & $\begin{array}{l}\text { HF erhöht, RR normal, normaler } \\
\text { Pulsdruck }\end{array}$ & $\begin{array}{l}\text { HF deutlich erhöht, RR unternormal, } \\
\text { geringer Pulsdruck }\end{array}$ & $\begin{array}{l}\text { Tachykardie, anschließend } \\
\text { Bradykardie, RR hypoton, } \\
\text { geringer Pulsdruck }\end{array}$ \\
\hline Zentralnervensystem & ängstlich, reizbar, verwirrt & lethargisch, ungeordnete Schmerzreaktion & komatös \\
\hline Haut & kühl, marmoriert, verlängerte CRF & zyanotisch, deutlich verlängerte CRF & blass und kalt \\
\hline Urinausscheidung & (sehr) wenig & minimal & keine \\
\hline HF: Herzfrequenz; RR: Blutdruck; CRF: capillary refill & & \\
\hline
\end{tabular}

letzten Kindern) $\rightarrow$ daher pH beachten und Ventilation optimieren.

Kinder reagieren anders auf Hypovolämie als Erwachsene. Daher ist es die erste Herausforderung, zunächst eine hämodynamische Störung überhaupt zu erkennen (vgl. - Tab. 1 und 2). Bei der Substitution und Gewichtsbestimmung kann dann das Breslow Pediatric Emergency Tape helfen oder die Faustformel 2× Alter + $10=$ Gewicht $[\mathrm{kg}]$. Bei der Hämodynamik ist stets auch die Urinausscheidung zu beobachten.

\section{Ältere Schwerverletzte}

Der Anteil älterer Schwerverletzter hat in den vergangenen Jahren stetig zugenommen und steigt weiterhin mit ca. $2 \%$ jährlich. Gründe dafür sind die steigende Lebenserwartung in den Industrienationen der letzten Jahrzehnte und das höhere Aktivitäts- und Mobilitätslevel der älteren Menschen. Während sich die Letalität unabhängig von der Verletzungsschwere bei älteren Traumapatienten entwickelt, zeigt sich eine deutlich steigende Letalität dieser Patienten zwischen dem 5. und 6. Lebensjahrzehnt. Diese Patientengruppe weist höhere Komplikationsraten, eine längere Krankenhausverweildauer und einen höheren Ressourcenverbrauch auf. Sie erleiden im Vergleich zum jüngeren Kollektiv unterschiedliche Unfallmechanismen (mehr Niedrigenergietraumata) bei gleich hoher Verletzungsschwere und ein anderes Verletzungsmuster (mehr schwere Schädel-Hirn-Traumata, weniger Abdominal- und Extremitätentraumata). Das Manage- ment sowohl in der Präklinik als auch im Schockraum ist bei älteren Schwerverletzten deutlich zurückhaltender. Trotz eines GCS $\leq 8$ (Glasgow Coma Scale) werden weniger ältere Traumapatienten intubiert, sie erhalten weniger Volumenzufuhr, kommen aber häufiger im dekompensierenden Schock im Schockraum an. Seltener erhalten Sie eine CT-Untersuchung und sie werden seltener operativ versorgt, trotz formaler Indikation bei SchädelHirn-Trauma, Beckenfrakturen und Extremitätenverletzungen. Ihre Krankenhausletalität ist höher als die der unter 50-Jährigen. Daher muss das Management älterer Schwerverletzter überdacht werden und das eher zurückhaltende Therapiekonzept in ein eher aggressiveres Konzept geändert werden. Wichtig ist jedoch auch die Berücksichtigung eines ggf. vorliegenden Patientenwillens und allgemeiner ethischer Grundlagen.

\section{Ertrinkungsunfälle}

Für Ertrinkungsunfälle, bei denen der Patient präklinisch intubiert und beatmet werden muss, wurde wie im Schockraumalarmierungsschema (vgl. > Abb. 1 und $\mathbf{3}$ b) eine eigene Alarmierungsstufe eingeführt. Der Hauptgrund dafür war, dass diesen Patienten - also nicht den „beinahe Ertrunkenen“ - eine möglichst sofortige Etablierung einer Herz-Lungen-Maschine (HLM) zum kontrollierten Aufwärmen und Stabilisieren des kardiorespiratorischen Systems zukommen soll. Dafür werden zur Schockraumalarmierung „Blau“ der Kardiotechniker sowie der Oberarzt der Thorax-Herz-Gefäßchirurgie im Rufdienst mobilisiert. Zusätzlich wird eine Bronchoskopie im 

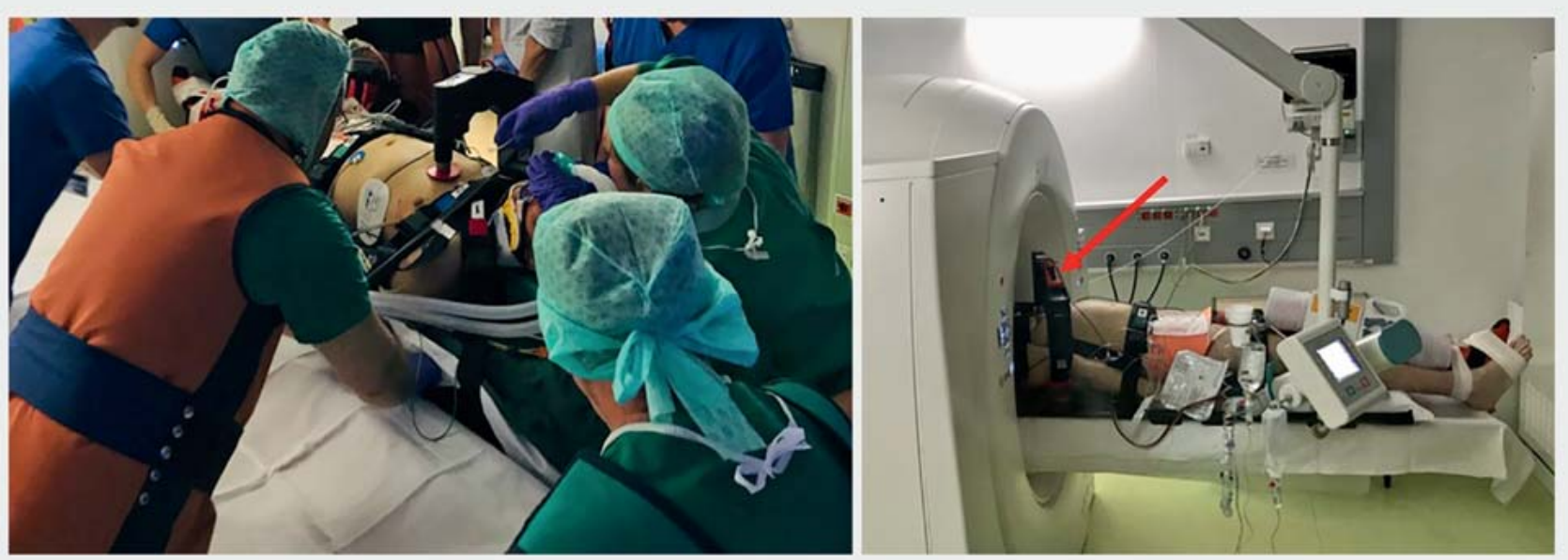

- Abb. 8 Verwendung des corpuls cpr $^{\circledR}$ bei der Durchführung eines Ganzkörper-CTs.

Schockraum durchgeführt. Bei signifikanter Hypothermie und (Z.n.) kardiopulmonaler Reanimation wird bereits vor Ankunft des Patienten die HLM hochgefahren. Auch wenn nicht zwingend ein Unfallgeschehen vorliegen muss, bleibt der Unfallchirurg Traumaleader, um das Schockraummanagement zu führen und ggf. Zeiten bis Eintreffen der Rufdienste zu überbrücken. Die Indikation zur Etablierung der HLM oder weiterführende Maßnahmen werden dann im gesamten Team konsentiert.

\section{Schwerverletzte unter laufender mechanischer kardiopulmonaler Reanimation}

Ein Schwerverletzter unter laufender kardiopulmonaler Reanimation (Schockraumalarmierungsstufe Rot, vgl. - Abb. 1) wird stets mit Oberarztteam im Schockraum erwartet. Bei diesen Patienten muss die Fortführung und die Dauer der Maßnahmen engmaschig reevaluiert werden. Allein zur Fortführung der Reanimation sollte die Teamstärke ausreichend groß sein. Seit Kurzem steht mit dem „corpuls $\mathrm{cpr}^{\circledR \text { « }}$ ein Gerät zur Verfügung, das die Fortführung der mechanischen Reanimation auch während der CT-Diagnostik ermöglicht (vgl. > Abb.8). Es muss jedoch dem Team bewusst sein, dass die Aussagen, die aus dem CT unter laufender mechanischer Reanimation erhoben werden können, sehr limitiert sind:

- Native Untersuchungen von Kopf bis mittlere HWS sind mit hoher diagnostischer Qualität möglich.

- Native Untersuchung von Thorax, Abdomen, unterer HWS bis LWS sind in guter bis mäßiger diagnostischer Qualität nur unter Gerätestillstand möglich.

- Ein Polytraumscan ist generell nur in Nativtechnik sinnvoll.

Es können damit nur limitierte Aussagen und Diagnosen gestellt werden. Die Fragestellung zur Durchführung eines CTs unter laufender Reanimation sollte daher limi- tiert sein auf wegweisende Aussagen zur Ursache der Reanimationspflichtigkeit und kritischer Prüfung, ob ein Fortsetzen der Maßnahmen weiterhin sinnvoll ist. Im vorliegenden Kollektiv werden ca. 3,5\% der über den Schockraum aufgenommenen Patienten unter laufender Reanimation eingeliefert oder sie werden unmittelbar nach Ankunft reanimationspflichtig. Diese weisen eine Krankenhausletalität von 74\% auf. Die Durchführung des CTs unter laufender mechanischer Reanimation ermöglicht es evtl., diese hohe Letalitätsrate zu senken, da nun CT-morphologische Befunde erhoben werden können und ggf. eine gezielte Therapie unmittelbar eingeleitet werden kann.

\section{Schlussfolgerung}

Das Schockraummanagement ist eine interdisziplinäre Teamleistung, die geprägt ist von klarer Kommunikation und eintrainierten Algorithmen. Der Schockraum ist das Bindeglied zwischen präklinischer Initialstabilisierung und definitiver Therapie. Dazu sind die vollumfängliche Befunderhebung und das Erkennen von sich ändernden pathologischen Dynamiken essenziell. Die Teamleistung besteht darin, diese komplexen Anforderungen parallel interdisziplinär und interprofessionell durch jeweilige Expertenteams abzuarbeiten und dem Gesamtteam zuzuarbeiten. Dabei kommt dem Leiter dieses Schockraummanagements (Traumaleader oder Führungsgruppe) die essenzielle Rolle zu, die Kommunikationsstrukturen in diesem Konstrukt zusammenzuführen und dem gesamten Team alle Informationen und Befunde mitzuteilen. Team-Time-out und Zeitpunkte für gemeinsame Evaluation der aktuellen Lage sind förderlich, um das Management von Schwerverletzten möglichst effizient zu gestalten. 


\section{Prognose und Ausblick}

Maßnahmen, die das Schockraummanagement positiv beeinflussen können sind

1. Team-Training,

2. Kurse zu Interpersonal Competence (z.B. der Deutschen Gesellschaft für Orthopädie und Unfallchirurgie in Kooperation mit Lufthansa Aviation Training) und

3. Qualitätszirkel, in denen neue Algorithmen und Geräte (z. B. corpuls cpr) kritisch gemeinsam bewertet und anschließend gezielt eingesetzt werden können.

In einer sich ändernden Krankenhaus- und Rettungsdienstlandschaft wird den Notfallaufnahmen - so auch dem Schockraum - eine immer wichtigere Aufgabe als Bindeglied und Auffangort für möglicherweise zunehmend eher untertriagierte Patienten zukommen. Auch im Falle des Massenanfalls von Verletzten (MANV) oder gar einer Terrorlage wird das Schockraummanagement durch die Versorgung der Schwerstverletzten mit einer besonderen Verantwortung konfrontiert sein. In diesen Lagen wird die Individualmedizin in den Hintergrund treten und das reine Überleben im Vordergrund stehen. Derartige Situationen kann nur ein gut geschultes und erprobtes Team bewältigen.

\section{Interessenkonflikt}

Die Autorinnen/Autoren geben an, dass kein Interessenkonflikt besteht.

\section{Autorinnen/Autoren}
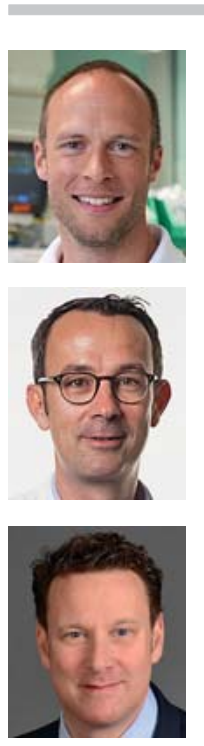

\section{Christopher Spering}

Dr. med., Oberarzt, Klinik für Unfallchirurgie, Orthopädie und Plastische Chirurgie, Universitätsmedizin Göttingen

\section{Stephan Sehmisch}

Prof. Dr. med., Leitender Oberarzt, Klinik für Unfallchirurgie, Orthopädie und Plastische Chirurgie, Universitätsmedizin Göttingen

\section{Wolfgang Lehmann}

Univ. Prof. Dr. med., Direktor der Klinik, Klinik für Unfallchirurgie, Orthopädie und Plastische Chirurgie, Universitätsmedizin Göttingen

\section{Korrespondenzadresse}

\section{Dr. med. Christopher Spering}

Oberarzt

Klinik für Unfallchirurgie, Orthopädie und Plastische Chirurgie Universitätsmedizin Göttingen

Robert-Koch-Straße 40

37099 Göttingen

Tel.: 0551/39-62188

christopher.spering@med.uni-goettingen.de

\section{Literatur}

[1] Spering C, Roessler M, Kurlemann T et al. Optimierte Ressourcenmobilisation und Versorgungsqualität Schwerstverletzter durch eine strukturierte Schockraumalarmierung. Unfallchirurg 2018; 121: 893-900. doi:10.1007/s00113-017-0447-6

[2] Deutsche Gesellschaft für Unfallchirurgie, Hrsg. Weißbuch Schwerverletztenversorgung. Empfehlungen zur Struktur, Organisation und Ausstattung stationärer Einrichtungen zur Schwerverletzten-Versorgung in der Bundesrepublik Deutschland. 3. Aufl. 2019. Im Internet: https://www.dgu-online.de/ fileadmin/published_content/5.Qualitaet_und_Sicherheit/ PDF/2019_DGU_Weissbuch_Schwerverletztenversorgung_ Vorabdruck.pdf; Stand: 02.12.2019

[3] American College of Surgeons. Resources for optimal care of the injured patient. 2014. Im Internet: https://www.facs. org/-/media/files/quality-programs/trauma/vrc-resources/ resources-for-optimal-care.ashx?la=en; Stand: 02.12.2019

[4] Brown R, Warwick J. Blue calls - time for a change? Emerg Med | 2001; 18: 289-292

[5] Calland JF, Xin W, Stukenborg G]. Effects of leading mortality risk factors among trauma patients vary by age. J Trauma Acute Care Surg 2013; 75: 501-505

[6] Cowley RA. The resuscitation and stabilization of major multiple trauma patients in a trauma center environment. Clin Med 1976; 83: 16-22

[7] Culemann U, Seekamp A, Riedel U et al. Interdisziplinäres Polytraumamanagement. Teil 2: Klinikaufnahme vital bedrohter traumatisierter Patienten. Notfall Rettungsmed 2003; 6: 573-579

[8] Handolin LE, Jääskeläinen J. Pre-notification of arriving trauma patient at trauma centre: a retrospective analysis of the information in 700 consecutive cases. Scand J Trauma Resusc Emerg Med 2008; 16: 15. doi:10.1186/1757-7241-16-15

[9] Häuser H, Bohndorf K, Rüter A. Der traumatologische Notfall im Schockraum. Analyse des Spektrums und des Zeitbedarfs der bildgebenden Diagnostik. Unfallchirurg 1998; 101: 129136

[10] Kanz K-G, Körner M, Linsenmaier U et al. Prioritätenorientiertes Schockraummanagement unter Integration des Mehrschichtspiralcomputertomographen. Unfallchirurg 2004; 107 : 937-944

[11] Knopp R, Yanagi A, Kallsen G et al. Mechanism of injury and anatomic injury as criteria for prehospital trauma. Ann Emerg Med 1988; 17: 895-902

[12] Kuehne CA, Ruchholtz S, Kaiser GM et al. Mortality in severely injured elderly trauma patients-when does age become a risk factor? World J Surg 2005; 29: 1476-1482

[13] Kühne CA, Ruchholtz S, Sauerland S. Personelle und strukturelle Voraussetzungen der Schockraumbehandlung Polytraumatisierter. Unfallchirurg 2004; 107: 851-861 
[14] Lillebo B, Seim A, Vinjevol OP et al. What is optimal timing for trauma team alerts? A retrospective observational study of alert timing effects on the initial management of trauma patients. J Multidiscip Healthc 2012; 5: 207-213

[15] Lovell MA, Mudaliar MY, Klineberg PL. Intrahospital transport of critically ill patients: complications and difficulties. Anaesth Intensive Care 2001; 29: 400-405

[16] Probst C, Hildebrand F, Frink $M$ et al. Erstversorgung Schwerstverletzter am Unfallort. Chirurg 2007; 78: 875-884

[17] Ruchholtz S, Nast-Kolb D, Waydhas $C$ et al. [Early mortality in polytrauma. A critical analysis of preventable errors]. Unfallchirurg 1994; 97: 285-291

[18] Smith DC, Chapital A, Burgess Uperesa BM. Trauma activations and their effects on non-trauma patients. J Emerg Med 2011; 41: 90-94

[19] Spering C, Lefering R, Bouillon B et al. It is time for a change in the management of elderly severely injured patients! An analysis of 126,015 patients from the TraumaRegister $D U^{\circledR}$. Eur J Trauma Emerg Surg 2019. doi:10.1007/s00068-019-012298
[20] Waydhas C, Baake M, Becker L et al. A consensus-based criterion standard for the requirement of a trauma team. World J Surg 2018; 42: 2800-2809

[21] Wurmb T, Balling $\mathrm{H}$, Frühwald $\mathrm{P}$ et al. [Polytrauma management in a period of change: time analysis of new strategies for emergency room treatment]. Unfallchirurg 2009; 112: 390-399

[22] Wutzler S, Lefering R, Wafaisade A et al. Aggressive operative treatment of isolated blunt traumatic brain injury in the elderly is associated with favourable outcome. Injury 2015; 46: 1706-1711

Bibliografie

DOI https://doi.org/10.1055/a-1023-5200

Online-publiziert 13.02.2020 | OP-JOURNAL 2020; 36: 18-29 (c) Georg Thieme Verlag KG Stuttgart · New York ISSN 0178-1715 